\title{
Treatment of Renal Disease: From Where Does the Opposite View Come?
}

\author{
Assem K. El-Sherif \\ Suez Canal University, Ismailia, Egypt
}

\section{Key Words}

Nephrology $\cdot$ Evidence-based medicine $\cdot$ Clinical practice

\begin{abstract}
Professor A.M. El Nahas has recently called for submitting articles concerned with the opposite view in nephrology practice. His call drew attention to several areas of controversy in the art. Also, it brought to mind a question about how an 'opposite view' emanates in a practice that ought to be based on experimental research and rigorous observational studies. This short input addresses the question of how nephrologists decide about management of their renal patients when the literature fails to grant an unequivocal answer to what they should do. It particularly focuses on the need to carefully read the existing literature and to appreciate its limitations. Also, it highlights the iterative nature of controversies in therapy of renal disease and how a controversy evolves into an 'opposite view'.
\end{abstract}

Copyright $\odot 2010$ S. Karger AG, Base

\section{Evidence}

In an old, well-structured analysis, Strippoli et al. [1] reported on the number and methodological quality of clinical trials published in the field of nephrology over 35 years (1966-2002). The number was fewer than in all other specialties of internal medicine and represented, for instance, less than $11 \%$ of randomized clinical trials published in cardiology over the same period. Also, the methodological quality of a random sample of the published nephrology trials was evaluated by two assessors and was not found to be better. Nevertheless, today no nephrologist has the capacity to look over all articles published in any one area of clinical nephrology and it remains prudent that nephrologists who crave to stay abreast of the state of the art have to adopt a highly selective approach in perusing the medical literature.

Evidence-based medicine (EBM) is simply defined as the sensible use of the best available scientific research and practices with proven effectiveness in daily medical decision-making about the care of individual patients. It is a life-long process since new evidence is being produced every day, which relentlessly modifies the clinical basis of diagnosis and therapy. In practice, EBM entails that you have to identify the needed information and reshape it into an answerable question. Then you search for the best available evidence with which you answer your question and carefully evaluate it. Critical appraisal is central to the practice of EBM - it involves systematic evaluation of methodological quality of the evidence for its validity and applicability.

\section{KARGER \\ Fax +4161306 1234 E-Mail karger@karger.ch} www.karger.com

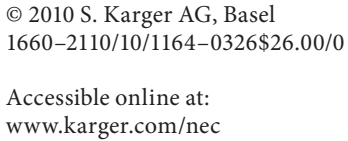

Prof. Assem K. El-Sherif

Department of Medicine, Suez Canal University

Ismailia (Egypt)

Tel. +20165464600

E-Mail akelsherif@gmail.com 
Uncritically accepting medical innovations is dangerous, whereas sophisticated armaments are obviously needed for the critical and highly selective approach to be efficient. For the busy clinician, systematic reviews and practice guidelines may provide attractive alternative options. However, systematic reviews are not always available to answer every question and they frequently lead to ambiguous recommendations, while guidelines customarily involve subjective value judgment. A systematic review of a small number of underpowered, poorly conducted, randomized controlled trials is clearly not better than one large, well-conducted, double- or triple-blinded trial. Besides, the algorithms that reduce patient care into a sequence of binary decisions often do injustice to the complexity of medicine and the parallel and iterative thought processes inherent in clinical judgment [2].

Today, the evidence on efficacy of mycophenolate mofetil for treatment of several forms of idiopathic glomerular diseases largely comes from descriptive studies. Only few randomized clinical trials and observational studies have been published so far [3-6] - all are underpowered and most of these are otherwise flawed in several aspects. Conclusions about the poor quality of mycophenolate mofetil studies have been explicitly drawn in meta-analyses and critical reviews done by few groups of researchers $[7,8]$, yet the fact is that many nephrologists are increasingly prescribing the drug for several forms of glomerular disease.

Being aware of the limitations facing research on glomerulonephritis, many nephrologists appear to rely on their own experience to synthesize the available information and employ that for resolving individual management decisions. The soundness of this approach is flimsy and, unsupported by the skills of critical apprais$\mathrm{al}$, can lead to menacing consequences. Indeed, the fundamental point about the limits of implementing research results is how we read it. Reading research is a complex skill that requires more than acquiring some knowledge of methods of designing a study or statistical manipulation of data. This concept was eloquently expressed years ago by Haines and Jones [9], though in a different context. They affirmed: 'It is unlikely that any single approach will be effective in ensuring the implementation of the findings from research. A more evaluative culture will require a shift in attitudes among health professionals to ensure that they rigorously analyze their own activities and accept the need for critical appraisal of evidence.'

\section{Evidence in Practice}

In clinical nephrology, glomerular diseases are named after their main pathological features and words such as mesangial, membranous, crescentic, endocapillary, and combinations of these are the nephrologists' daily jargon. Each pathological pattern presents to the clinician in different syndromic features and, on the other hand, each one of the presenting syndromes is linked to a variety of pathologies. Hence, it is no wonder that we fall into trouble when a patient with 'membranous nephropathy', or any other chronic glomerulonephritis, enquires about the fate of his own disease. It may take us a long time of serious nephrology practice before we appreciate that in nephrology, probably more than in any other specialty, we deal with a clinicopathological matrix of dynamic features rather than a well-defined single dimension. Frequently, this matrix divulges puzzles and is often full of holes.

Patients entitled to have 'idiopathic membranous nephropathy' may end up having complete spontaneous recovery, end-stage renal disease, or anything in between. The natural history of the disease reflects, in great part, its etiology/pathogenesis, which is largely unknown ${ }^{1}$, and it directly impacts treatment.

With some knowledge and little experience, most of us will acknowledge that membranous nephropathy is heterogeneous in nature and its treatment is largely empirical. Hence, we often respond to the patient's questions by referring to probabilities of different outcomes and different responses to treatment policies. The patient may further enquire about what determines these outcomes and what determines variability of response to therapy (predictors in our terminology). We tell him about what has been studied of these predictors so far and point out that each predictor is linked to any given outcome only with some calculable probability. Also, we may tell him that pathological and clinical features likely interact in a complex fashion.

Because most of the assumingly independent probability values (predictive values) are not very high, we sequentially sum few of these together following the bayesian model, so that we can tailor our judgment to the best

\footnotetext{
1 Neutral endopeptidase alloimmunization has been determined to cause neonatal membranous nephropathy, but this is a distinctive form of the disease reported in only a small subset of patients [10]. Also, the significance of detection of anti-PLA2R autoantibodies and its possible role in the pathogenesis of idiopathic membranous nephropathy in adults remain to be sufficiently established [11].
} 
for this particular patient. A knowledgeable, conscientious clinician may involve the patient in this exercise and may take him a step further helping him to decide, through a similar process of 'decision analysis', about his own choice of intervention. Ideally the patient is the one who can fix a 'utility' to each of the possible outcomes of management options and, helped out by the doctor's knowledge, is able to settle on one of the alternative options. An alkylating agent carries significant risks of reduced fertility and malignancy in the long term. Such risks will be differently weighed up by an 18-year-old female patient and a 65-year-old one. Hence, the two patients will assign different utility figures for this treatment, which eventually impact their management policies and prognosis.

\section{Experience and Evidence}

It is not true that doctors are conditioned to dislike mathematics. In fact, when exploring a diagnosis, any doctor decides about therapy or hypothesizes about a prognosis, and builds up his work on implicit stepwise calculation of probabilities. Sound and up-to-date knowledge of the relevant clinical research, beside attentive experience, determines how the doctor judges these probabilities. Methodologically, he applies roles of epidemiology to his clinical practice. This is the domain of clinical epidemiology which has been defined by Fletcher and Fletcher [12] as 'the science of making predictions about individual patients by counting clinical events in groups of similar patients and using strong scientific methods to ensure that the predictions are accurate'.

Evidence on efficacy of dialysis therapy has never come from a planned controlled study in which a number of uremic patients were assigned to receive the treatment while others were not. Early evidence came from a uniquely exceptional event of recovery of a dying uremic patient after dialysis was tried in the late 1940s. Today, similar evidence is difficult to come by and a 'randomized clinical trial' is the best available design to conclude about efficacy of a medical intervention. In this design, randomization balances confounding factors between treatment groups, allows for blinding and provides a robust basis for making scientific inferences. Yet, the inherent limitations in definition and classification of renal disease and the intrinsic difficulty in representing clinical reality by the available clinical trials create fundamental problems in extrapolating the results of these trials to the individual patient. In all clinical trials the num- bers of patients who participate are limited, their baseline characteristics at inception usually reflect a highly selective recruitment criterion, and the treatment periods are always much shorter than in regular management of a chronic kidney disease. Good clinical trials in nephrology are those reported by reviewers to have high levels of internal validity. However, external validity remains a major trouble in nephrology practice and the question of whether results of a given study are applicable to 'this particular patient' is a highly relevant one in that practice. The presence of co-morbidities, variation in disease severity and disease activity, peculiarities of the natural history of the patient's illness, the used concomitant therapies and the potential for development of adverse and side effects from treatment all are important factors that impact the decision of individualizing management policies. Here comes the experience which reflects competency and reasoning acquired through thoughtful clinical practice.

\section{Controversy and the Opposite View}

When competency and reasoning are integrated with broad analytical awareness of the available evidence, the harvest is sound individualization of patients' management. Shortage/defectiveness of evidence are ubiquitous in medicine and are the major source of disputes encountered in clinical practice. For example, because of the lack of well-designed randomized clinical trials, an explicit role for steroid therapy in the resolution of drug-induced interstitial nephritis remained uncertain. Earlier small studies suggested that steroid therapy enhances disease recovery but has no impact on prognosis. Common sense describes this as inconsistency, and indeed it is. Doctors held different views to explain the paradox created by these studies, but in practice they were divided into those who use steroids, once withdrawal of the offending medication fails to promptly restore kidney function, and others who question steroid use and indications suggested by experts for treatment of acute interstitial nephritis. This disagreement is classically referred to as a 'controversy' and remains a popular subject for sessions devoted to debatable areas in nephrology congresses. Both the pro- and the anti-steroid groups rely on deficiency of evidence to support their point of view. More recently there has been a shift towards early use of steroids in drug-induced acute interstitial nephritis. This shift was based on retrospective analysis of treatment data and on studies correlating clinical and pathological responses to steroid therapy. An 
update of treatment effects has strongly concluded that early steroid administration eradicates inflammatory infiltrates more rapidly and completely, and may thus be important to minimize subsequent chronic damage [13, 14].

Nevertheless, the controversy will continue to exist and new controversies will likely emerge, awaiting firm evidence to come from adequately powered randomized clinical trials on a homogeneous population of patients. Indeed, poor evidence frequently authorizes the appearance of biased articles and subsequently creates contro- versies. In addition, the term 'personalized medicine' has been badly employed in several ways. It should be redefined to speak of the integration between clinical reasoning and evidence-based medicine for the interest of management of the individual patient. The iterative nature of the above-mentioned quandaries argues against departure of controversies. As one controversy is solved, others are generated, but only when defective experience and reasoning abilities are combined with shallow or sketchy understanding of the available evidence, then a controversy may pile up to create an 'opposite view'.

\section{References}

1 Strippoli GF, Craig JC, Schena F: The number, quality, and coverage of randomized controlled trials in nephrology. J Am Soc Nephrol 2004;15:411-419.

-2 Woolf SH, Grol R, Hutchinson A, Eccles M, Grimshaw J: Clinical guidelines: potential benefits, limitations and harms of clinical guidelines. BMJ 1999;318:527-530.

-3 Branten AJ, du Buf-Vereijken PW, Vervloet M, Wetzels JF: Mycophenolate mofetil in idiopathic membranous nephropathy: a clinical trial with comparison to a historic control group treated with cyclophosphamide. Am J Kidney Dis 2007;50:248-256.

4 Dussol B, Morange S, Burtey S, Indreies M, Cassuto E, Mourad G, Villar E, Pouteil-Noble C, Karaaslan H, Sichez H, Lasseur C, Delmas Y, Nogier MB, Fathallah M, Loundou A, Mayor V, Berland Y: Mycophenolate mofetil monotherapy in membranous nephropathy: a 1-year randomized controlled trial. Am J Kidney Dis 2008;52:699-705.
Segarra A, Amoedo ML, Martinez Garcia JM, Pons S, Praga M, Garcia EI, Alonso JC Gascó JM, Pou L, Piera L: Efficacy and safety of 'rescue therapy' with mycophenolate mofetil in resistant primary glomerulonephritis - a multicenter study. Nephrol Dial Transplant 2007;22:1351-1360.

-6 Senthil Nayagam L, Ganguli A, Rathi M, Kohli HS, Gupta KL, Joshi K, Sakhuja V, Jha $\mathrm{V}$ : Mycophenolate mofetil or standard therapy for membranous nephropathy and focal segmental glomerulosclerosis: a pilot study. Nephrol Dial Transplant 2008;23:19261930.

7 Xu G, Tu W, Jiang D, Xu C: Mycophenolate mofetil treatment for IgA nephropathy: a meta-analysis. Am J Nephrol 2009;29:362367.

8 Cshiepatti A, Perna A, Zamora A, Giuliano GA, Braun N, Remuzzi G: Immunosuppressive treatment for idiopathic membranous nephropathy in adults with nephritic syndrome. Cochrane Database Syst Rev 2004; CD004293.

-9 Haines A, Jones R: Implementing findings of research: comment. BMJ 1994;309:412.
10 Debiec H, Guigonis V, Mougenot B, Decobert F, Haymann JP, Bensman A, Deschênes G, Ronco PM: Antenatal membranous glomerulonephritis due to anti-neutral endopeptidase antibodies. N Engl J Med 2002; 346:2053-2060.

11 Prunotto M, Carnevali ML, Candiano G, Murtas C, Bruschi M, Corradini E, Trivelli A, Magnasco A, Petretto A, Santucci L, Mattei S, Gatti R, Scolari F, Kador P, Allegri L, Ghiggeri GM: Autoimmunity in membranous nephropathy targets aldose reductase and SOD2. J Am Soc Nephrol 2010;21:507-519.

12 Fletcher RW, Fletcher SW: Clinical Epidemiology: The Essentials, ed 4. Philadelphia, Lippincott Williams \& Wilkins, 2005, p 3.

13 González E, Gutiérrez E, Galeano C, Chevia C, de Sequera P, Bernis C, Parra E, Delgado R, Sanz M, Ortiz M, Goicoechea M, Quereda C, Olea T, Bouarich H, Hernández Y, Segovia $B$, Praga M: Early steroid treatment improves the recovery of renal function in patients with drug-induced acute interstitial nephritis. Kidney Int 2008;73:940-946.

14 Praga M, González E: Acute interstitial nephritis. Kidney Int 2010;77:956-961. 